\title{
A Novel Account of the 'Forgotten Disease'
}

https://doi.org/10.47210/bjohns.2020.v28i2.324

Saikat Samaddar, ${ }^{1}$ Titas Kar, ${ }^{2}$ Rahul Sarkar, ${ }^{3}$ Saumendra Nath Bandyopadhyay ${ }^{3}$

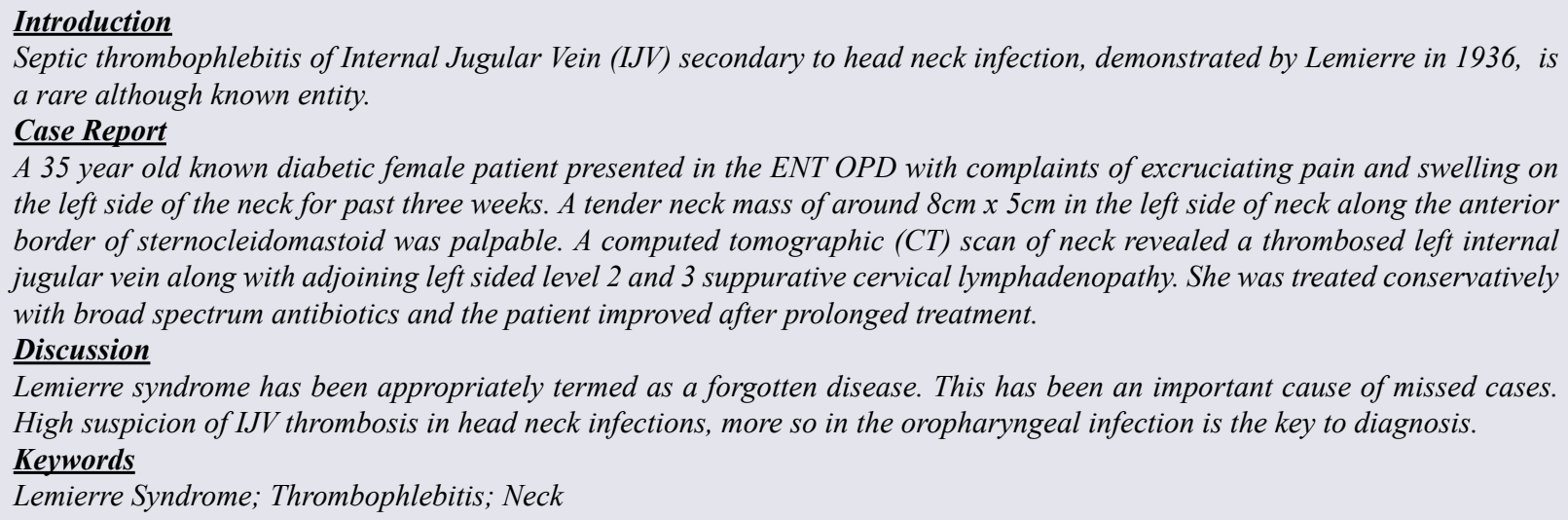

Septic thrombophlebitis of Internal Jugular Vein (IJV) secondary to head neck infection, demonstrated by Lemierre in 1936, is a rare although known entity.

Case Report

A 35 year old known diabetic female patient presented in the ENT OPD with complaints of excruciating pain and swelling on the left side of the neck for past three weeks. A tender neck mass of around $8 \mathrm{~cm} \times 5 \mathrm{~cm}$ in the left side of neck along the anterior border of sternocleidomastoid was palpable. A computed tomographic (CT) scan of neck revealed a thrombosed left internal jugular vein along with adjoining left sided level 2 and 3 suppurative cervical lymphadenopathy. She was treated conservatively with broad spectrum antibiotics and the patient improved after prolonged treatment.

Discussion

Lemierre syndrome has been appropriately termed as a forgotten disease. This has been an important cause of missed cases. High suspicion of IJV thrombosis in head neck infections, more so in the oropharyngeal infection is the key to diagnosis. Kevwords

Lemierre Syndrome; Thrombophlebitis; Neck

0 eptic thrombophlebitis of Internal Jugular Vein (IJV) secondary to head neck infection is a rare although known entity. Demonstrated by Lemierre in 1936, the disease still continues to have fatal natural course. ${ }^{1}$ In the past few decades there has been a significant decrease in the incidence of the disease, probably due to the advent of a vast spectrum of antibiotics. ${ }^{2}$ Lemierre syndrome affects mainly healthy people in the second and third decades of life. ${ }^{3}$

Fusobacterium necrophorum, a Gram-negative nonspore-forming obligate anaerobe is the main culprit

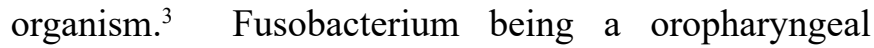
commensal leads to Lemierre's syndrome following oropharyngeal and dental infections which accounts for $85 \%$ of the cases of the disease. ${ }^{4}$ This article describes one such rare case.

\section{Case Report}

A 35 year old known diabetic female patient presented in the ENT OPD in a tertiary care hospital of Eastern
India with complaints of excruciating pain and swelling on the left side of the neck for past three weeks. She had fever with highest recordable temperature of around $103^{\circ} \mathrm{F}$. There was associated complaint of lethargy. The patient was a follow up case in the same department for chronic rhinosinusitis. She used to present with recurrent episodes of nasal blockage, cough and cold, headache, running nose. On physical examination the patient was febrile, tachypnoeic, tachycardic. A neck mass of around

1 - Department of Otorhinolaryngology and Head Neck Surgery, Purulia Government Medical College and Hospital, Purulia

2 - Department of Otorhinolaryngology and Head Neck Surgery, Institute of Postgraduate Medical Education and

Research, Institute of Otorhinolaryngology and Head Neck Surgery, Kolkata

3 - Department of Otorhinolaryngology and Head Neck Surgery, Medical College and Hospital Kolkata

\section{Corresponding author:}

Dr Titas Kar

email: titaskar@yahoo.com 


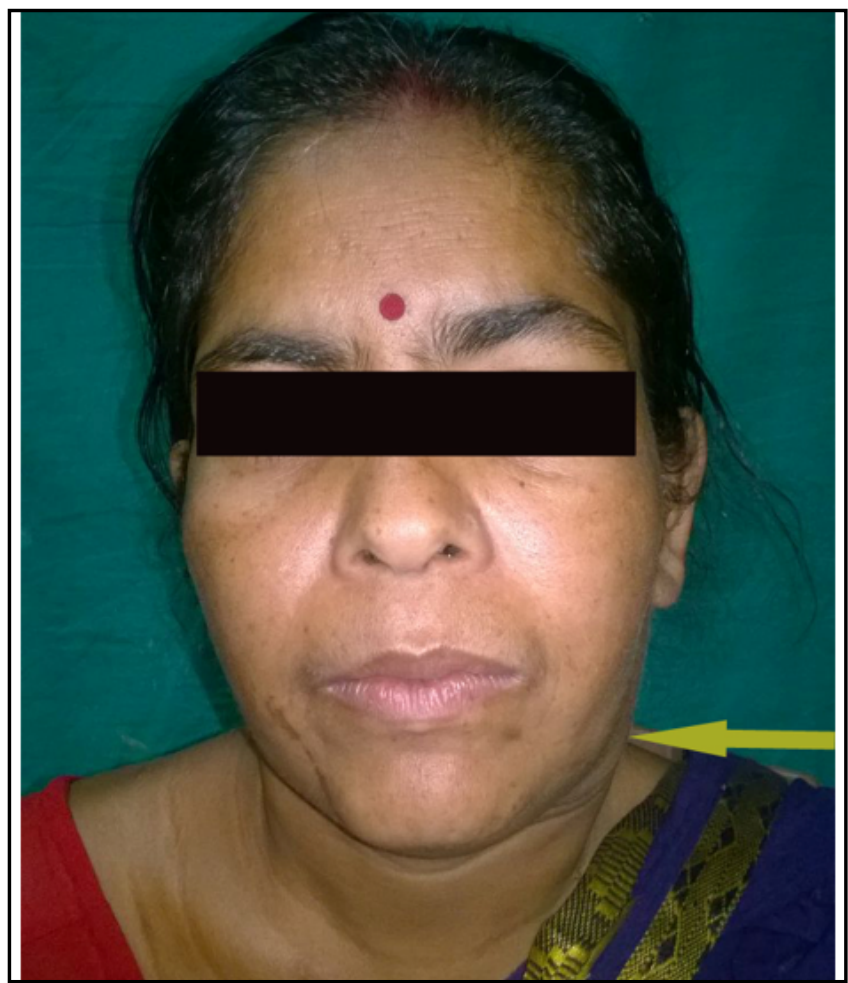

Fig. 1. Arrow points to the mass on the left side of the neck

$8 \mathrm{~cm} \times 5 \mathrm{~cm}$ in the left side of neck along the anterior border of sternocleidomastoid was palpable.(Fig.1) It was tender, local temperature was raised, firm in consistency, margins ill-defined and fixed to underlying structures. Nasal examination revealed mucopus in the right nasal cavity.

The patient was admitted in the indoor unit of the same department and was managed conservatively. Baseline work up revealed uncontrolled glycaemic status. A computed tomographic (CT) scan of neck (Fig. 2) revealed a thrombosed left internal jugular vein along with adjoining left sided level 2 and 3 suppurative cervical lymphadenopathy. CT scan of the paranasal sinuses (Fig. 3) showed hypodense shadows in the ethmoids, right sphenoid sinuses and a non contrast enhancing mass extending from the right nasal cavity into the nasopharynx. CT scan of chest did not demonstrate any significant abnormality. In Colour Doppler study, extracranial part of left IJV showed echogenic thrombus in its lumen with no flow, suggestive of IJV thrombosis. Haematological investigations showed increased leucocyte count with polymorphonuclear leucocytosis.

A blood culture specimen in both aerobic and anaerobic medium was collected aseptically and sent for microbiological assessment. Broad spectrum antibiotics were started in the form of injection Clindamycin. The culture sensitivity report obtained thereafter failed to demonstrate any significant pathological flora. So the same antibiotics were continued for three weeks. In consultation with cardio thoracic and vascular surgeons anticoagulant therapy was started in the form of heparin and warfarin. Heparin administration was tapered off in the next three days. Warfarin was continued for two weeks. A detailed recording of the patient's haemo-

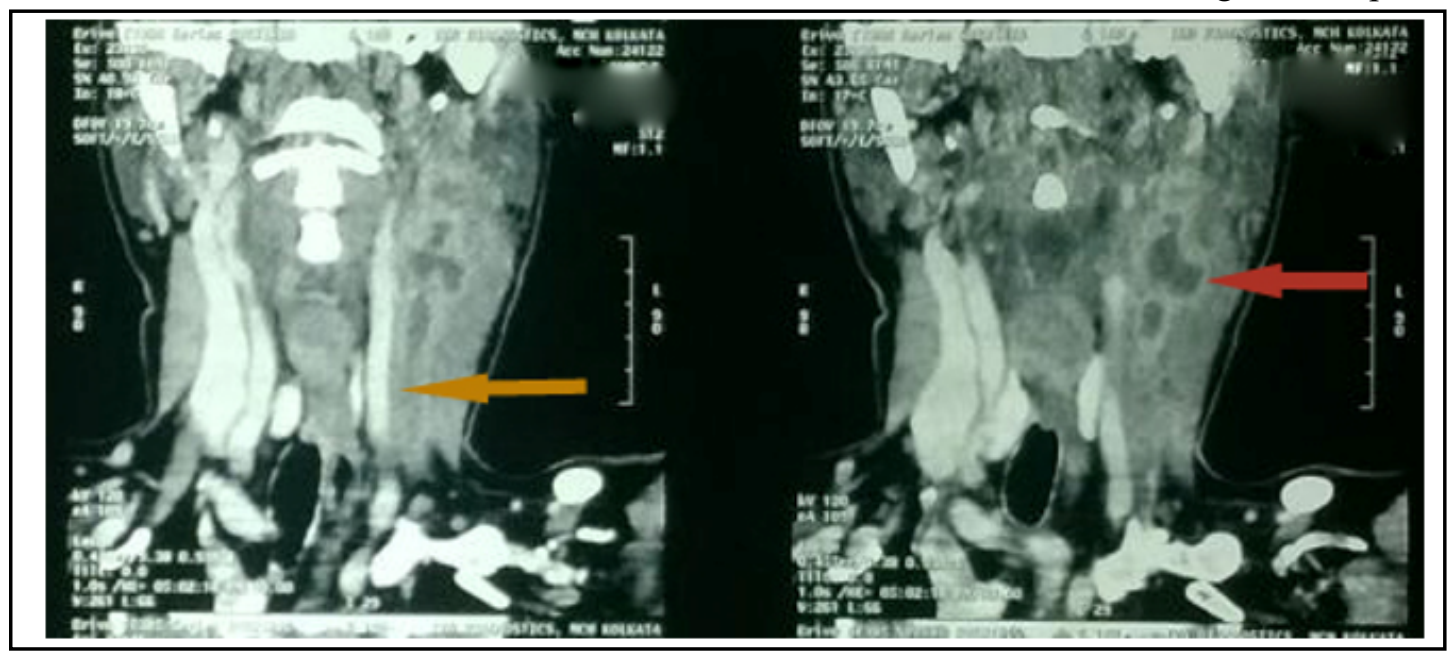

Fig. 2. CT scan of neck. Yellow arrow points to the thrombosed left internal jugular vein. Red arrow points to adjoining left sided level 2 and 3 suppurative cervical lymphadenopathy with central necrosis 


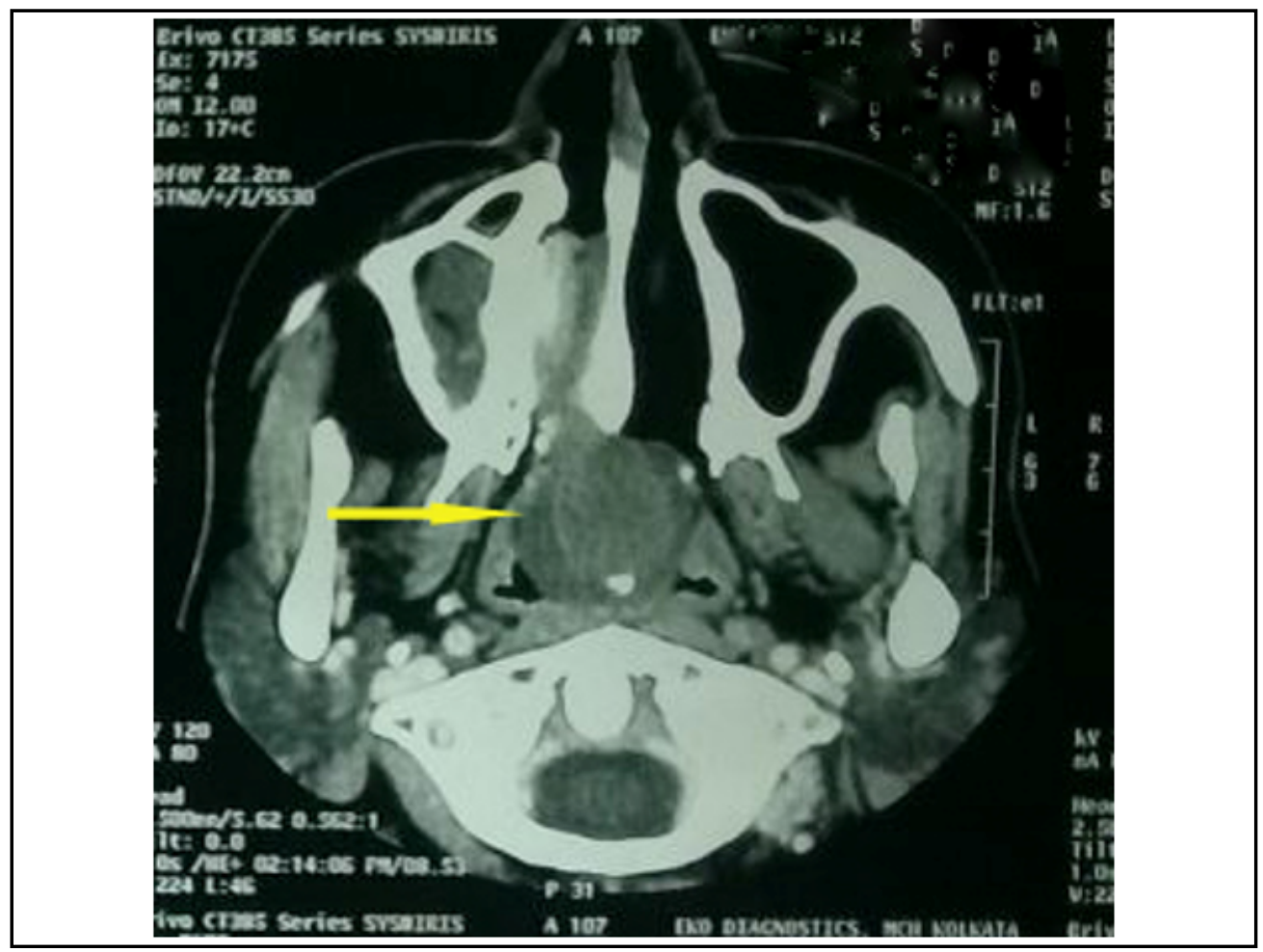

Fig. 3. Non contrast enhancing mass in left nasal cavity extending to nasopharynx

dynamic status was ensured. The stay in hospital was uneventful with the patient getting discharged in afebrile state with subsided neck swelling, after 3 weeks of injectable antibiotic administration. No surgical intervention had to be resorted to for the neck mass. Intranasal corticosteroid spray and oral antibiotics consisting of amoxycilin clavulanate was prescribed for three weeks along with probiotic capsules. Subsequent diagnostic nasal endoscopies showed that the nasal passage had cleared considerably due to reduction in size of the nasal polyp. Endoscopic excision of the nasal polyp was planned two months later after optimization of the glycemic status of the patient but she did not give consent for surgery and opted for medical management of rhinosinusitis.

\section{Discussion}

Septic thrombophlebitis of IJV secondary to head neck infection was taken as a case definition to diagnose Lemierre Syndrome. ${ }^{1}$
Postanginal sepsis was the term used by many authors in the first 40 years of the 20th century. ${ }^{5,6}$ Vogel and Boyer in 1980 first included "Lemierre" in the title of their case report. ${ }^{7}$ Shannon et al. in 1983 have been the first authors to use the term "Lemierre syndrome", in the title of their paper. ${ }^{8}$

Differences exist as to whether Lemierre syndrome relates to infection arising in the oropharynx caused by F. necrophorum or should it include infections arising in any site other than the oropharynx, or infections by non Fusobacterium group. ${ }^{9} \quad$ F. necrophorum although thought to be an unusual commensal of the oral flora can exist in other sites of the body. Lemierre reported that infection could arise from the gastrointestinal tract, the female genital tract, or the urinary tract. ${ }^{10}$ Alston et al identified 21 published cases of $F$. necrophorum in the United Kingdom, of which six were directly related to tonsillar infection and would be compatible with Lemierre syndrome, seven infections arose from the female genital tract, including three cases that were postabortion or postpartum. ${ }^{10}$ 
There is dearth of data regarding the presence of this bacterium in the lymphoid organs other than the Waldeyer's ring. This case reports IJV thrombosis secondary to suppurative lymphadenitis of neck which in turn is secondary to an acute exacerbation of a chronic rhinosinusitis in an immunocompromised patient. There had been no case reports of IJV thrombosis from suppurative lymphadenitis as of now.

IJV thrombophlebitis at the early stage might be asymptomatic, so there is considerable dependence on imaging techniques. Radiological demonstration of IJV thrombosis might be the first evidence to suggest the diagnosis of Lemierre syndrome. ${ }^{11}$ Extensive thrombophlebitis may be an indication for anticoagulation therapy or even surgical ligation. The standard method of diagnosis of deep venous thrombosis is catheter venography. However, this is invasive and carries the risk of propagating a septic process. ${ }^{12}$ So it is rarely performed. Ultrasound scanning has been advocated for imaging jugular venous thrombosis. The technique is rapid, low cost, and noninvasive. But ultrasound cannot image beneath the clavicle or the mandible. Ultrasound may fail to indicate small thrombus. So it's sensitivity is impaired. ${ }^{12}$ Several studies have demonstrated failure of ultrasonography to demonstrate internal jugular vein thrombosis that was readily demonstrable by contrast CT. ${ }^{13}$ It is, however, of particular value for serial scanning after initial imaging.

Blood culture remains the standard for identification of Fusobacterium. But reliable identification to species level, additional tests are required. F. necrophorum is the only Fusobacterium sp. that ferments lactate to propionate, a fact that that is used for confirmation of $\mathrm{F}$. necrophorum by gasliquid chromatography. However, for most routine laboratories, confirmation would probably be best achieved by referral of significant isolates, such as from blood culture, to a reference laboratory. ${ }^{14}$ Non identification of Fusobacterium in blood culture in our case causes a diagnostic dilemma. It makes us ponder whether Lemierre syndrome can be caused by other pathogens. According to Riodan et al. the evidence is inconclusive as to whether F. necrophorum is the sole cause of Lemierre's syndrome. ${ }^{14}$

Since Lemierre Syndrome involves IJV thrombosis and abscess lacunae in the lungs the antibiotic choice and regimen has to be appropriately tailored. Amoxicillin and clavulanic acid or clindamycin, together with metronidazole remain the first line of defense before further wide spectrum antibiotics are used, such as vancomycin or imipenem. ${ }^{15}$ Clindamycin is not susceptible to the "Eagle effect" as is penicillin. Empirical use of clindamycin has thus been advocated in severe infections. ${ }^{16}$ The use of anticoagulants has been controversial, and no controlled studies exist.

Other treatment modalities include IV immunoglobulin, activated protein $\mathrm{C}$, and hyperbaric oxygen in order to improve the immune response against the bacterial super-antigens. This modalities are still in experimental stage..$^{17,18}$

\section{Conclusion}

Lemierre Syndrome has been appropriately termed as a forgotten disease. This has been an important cause of missed cases. High suspicion of IJV thrombosis in head neck infections, more so in the oropharyngeal infection is the key to diagnosis. Contrast enhanced CT of the neck is a tool to identify the thrombus. Demonstration of causative organism should not restrict the clinician to start antibiotic therapy. Clindamycin is the empirical antibiotic of choice. Long term antibiotic regimen is advocated. Need for anticoagulant therapy is debated. Early identification and appropriate intervention reduces the mortality.

\section{References}

1. Lemierre A. On certain septicaemias due to anaerobic organisms. Lancet. 1936; 227:701-3

2. McMullan R, McConville C, Clarke JC, Adams DA, Hedderwick S. Lemierre syndrome: Remember the forgotten disease. Ulster Med J. 2004;73:123-5

3. Righini CA, Karkas A, Tourniaire R, et al. Lemierre syndrome: study of 11 cases and literature review. Head Neck 2014; 36(7):1044-51. doi: 10.1002/hed.23410

4. Moore BA, Dekle C, Werkhaven J. Bilateral Lemierre's syndrome: A case report and literature review. (238-40). Ear Nose Throat J. 2002; 81:234-6

5. Abt, I. A. 1932. Postanginal sepsis. J Pediatr. 1:8-15

6. Boharas, S. Postanginal sepsis. Arch. Intern. Med. 1943; 71:844-53 
7. Vogel, LC, Boyer KM. Metastatic complications of Fusobacterium necrophorum sepsis. Am J Dis Child 1980; $134: 356-8$

8. Shannon GW, Ellis CV, Stepp WP. Oropharyngeal Bacteroides melaninogenicus infection with septicemia: Lemierre's syndrome. J Fam Pract. 1983; 16:159-66

9. Chirinos JA, Lichtstein DM, Garcia J, Tamariz LJ. The evolution of Lemierre syndrome. Report of 2 cases and review of the literature. Medicine 2002; 81:458-65

10. Alston JM. Necrobacillosis in Great Britain. Br Med J. 1955; 1524-8

11. Screaton NJ, Ravenel JG, Lehner PJ, Heitzman ER, Flower CDR. Lemierre syndrome: forgotten but not extinct - report of four cases. Radiology 1999; 213:369-74

12. Braun IF, Hoffman JC Jr, Malko JA, Pettigrew RI, Daniels W, Davis PC. Jugular venous thrombosis: MR imaging. Radiology $1985 ; 157: 357-60$
13. Hong P, MacCormick J, Lamothe A, Corsten M. Lemierre syndrome: presentation of three cases. J. Otolaryngol. 2005; 34:352-8

14. Riordan T. Human infection with Fusobacterium necrophorum (Necrobacillosis), with a focus on Lemierre's syndrome. Clin Microbiol Rev. 2007; 20:622-59

15. Hughes CE, Spear RK, Shinabarger CE, Tuna IC. Septic pulmonary emboli complicating mastoiditis: Lemierre's syndrome revisited. Clin Infect Dis. 1994; 18:633-5

16. Pillai A, Thomas S, Williams C. Clindamycin in the treatment of group $\mathrm{G}$ beta-haemolytic streptococcal infections. J Infect. 2005; 51:e207-11

17. Hodgson R, Emig M, Pisarello J. Hyperbaric oxygen (HBO2) in the treatment of Lemierre syndrome. Undersea Hyperb Med. 2003; 30:87-91

18. Tewfik TL, Husein M, Shapiro RS, Oudjhane K. Lemierre syndrome in an immunocompromised patient. Int $\mathbf{J}$ Pediatr Otorhinolaryngol. 1999; 51:195-9. 